Supporting Information for

\title{
The Chemical Design Principles for Axis-dependent Conduction Polarity
}

Yaxian Wang ${ }^{\S}$, Karl G. Koster ${ }^{\dagger}$, Andrew M. Ochs ${ }^{\dagger}$, Michael R. Scudder ${ }^{\dagger}$, Joseph P. Heremans ${ }^{\ddagger}, \zeta$, Wolfgang Wind ${ }^{\S, \zeta}$, Joshua E. Goldberger ${ }^{\dagger *}$

${ }^{\S}$ Department of Materials Science and Engineering, The Ohio State University, Columbus, $\mathrm{OH} 43210$

${ }^{\dagger}$ Department of Chemistry and Biochemistry, The Ohio State University, Columbus, $\mathrm{OH} 43210$

${ }^{\ddagger}$ Department of Mechanical and Aerospace Engineering, The Ohio State University, Columbus, $\mathrm{OH} 43210$

'Department of Physics, The Ohio State University, Columbus, OH 43210

1. Mathematical derivation of wavefunction at $K$ point in a hexagonal unit cell

As the electronic structure of hexagonal lattices is often very complex, here we derive how the phases of adjacent molecular orbitals change in a periodic hexagonal lattice for different reciprocal lattice wavevectors across the Brillouin zone.

First, we mathematically define the real space hexagonal lattice of a single honeycomb layer. Our unit cell consists of an array of hexagons, for instance, the CuP layer in BaCuP. The distance between the center of two adjacent hexagons is assigned a value of lattice constant " $a$ " as seen in Figure S1. The distance between the middle of two hexagons in the horizontal direction is equal to $a \sqrt{3}$.

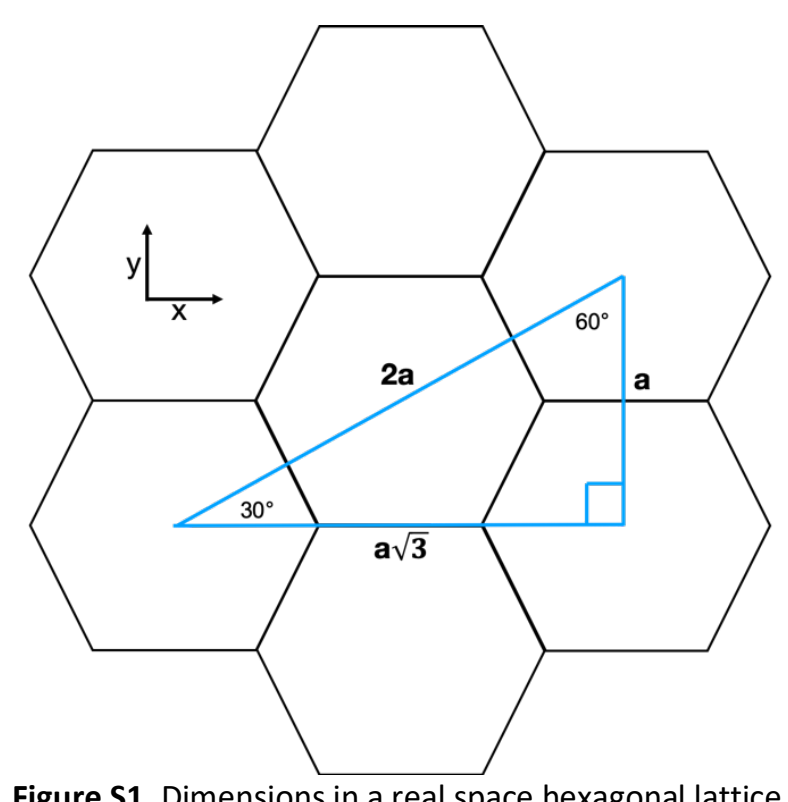

Figure S1. Dimensions in a real space hexagonal lattice.

The unit cell for this hexagonal structure is shown in Figure S2. The edges of this unit cell can be described by the following vectors, $\overrightarrow{a_{1}}$ and $\overrightarrow{a_{2}}$ :

$\overrightarrow{a_{1}}=\frac{a \sqrt{3}}{2} \hat{x}+\frac{a}{2} \hat{y} \quad \overrightarrow{a_{2}}=\frac{a \sqrt{3}}{2} \hat{x}-\frac{a}{2} \hat{y}$ 
In the hexagonal system, atoms are found at points $c_{1}$ and $c_{2}$. One of these atoms is phosphorus while the other is copper. It does not matter which atom is which, as long as its placement is consistent among all unit cells. The vectors from the origin of the unit cell to each of these points are:

$\overrightarrow{c_{1}}=\frac{a}{\sqrt{3}} \hat{x}+0 \hat{y} \quad \overrightarrow{c_{2}}=\frac{2 a}{\sqrt{3}} \hat{x}+0 \hat{y}$

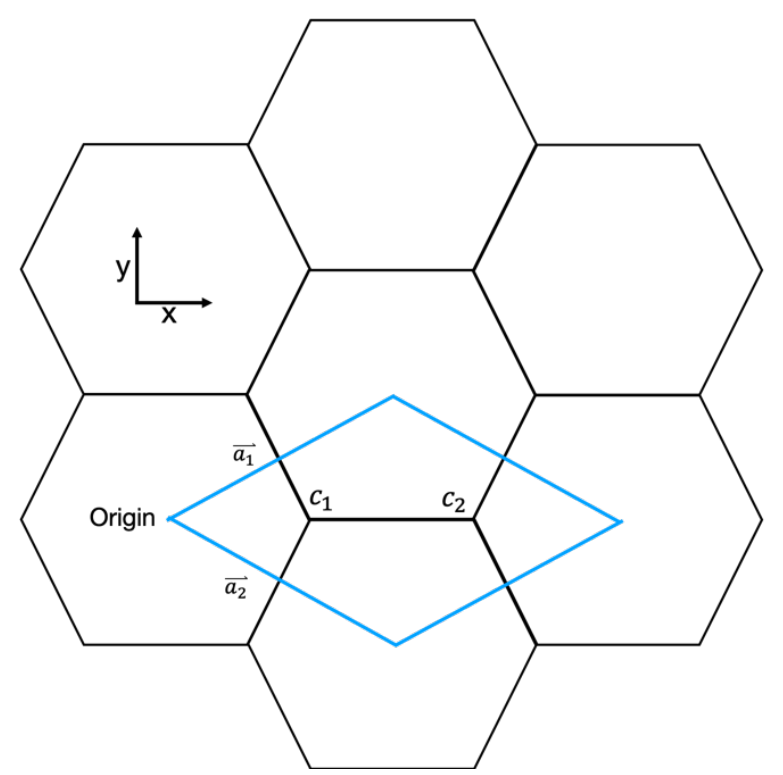

Figure S2: The unit cell in a hexagonal system.

Now consider the transition from real space to reciprocal space. Figure S3 shows a hexagonal lattice in reciprocal space. The reciprocal space lattice still has a hexagonal unit cell, but is rotated by $30^{\circ}$ compared to the real space unit cell, Figures S1 and S2. In this transition, the reciprocals of the real space vectors $\overrightarrow{a_{1}}$ and $\overrightarrow{a_{2}}$ are multiplied by $\pi$ to obtain the reciprocal space lattice vectors $\overrightarrow{b_{1}}$ and $\overrightarrow{b_{2}}$

$\overrightarrow{b_{1}}=\frac{2 \pi}{\sqrt{3} a} \hat{k}_{x}+\frac{2 \pi}{a} \hat{k}_{y} \quad \overrightarrow{b_{2}}=\frac{2 \pi}{\sqrt{3} a} \hat{k}_{x}-\frac{2 \pi}{a} \hat{k}_{y}$ 


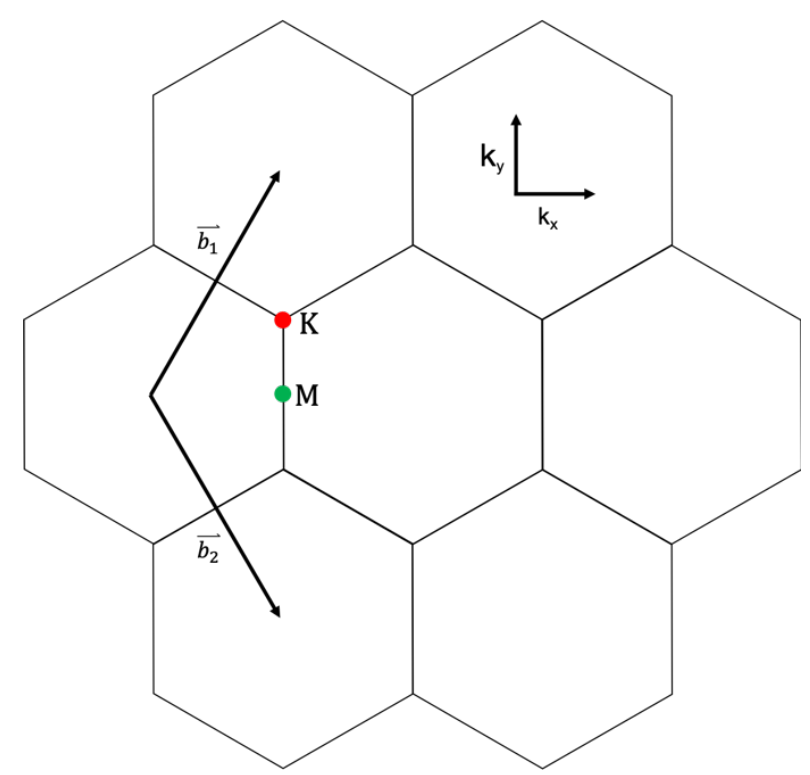

Figure S3. Reciprocal lattice vectors and symmetry points $\mathrm{K}$ and $\mathrm{M}$ in the hexagonal system.

Finally, the first Brillouin zone is defined by the symmetry points $K$ and $M$ in the reciprocal space unit cell. Using some simple trigonometry, these points can be determined to be:

$\mathrm{K}=\frac{2 \pi}{\sqrt{3} a} \hat{k}_{x}+\frac{2 \pi}{3 a} \hat{k}_{y} \quad \mathrm{M}=\frac{2 \pi}{\sqrt{3} a} \hat{k}_{x}+0 \hat{k}_{y}$

This information can be used to calculate the relative phases of identical atoms in adjacent unit cells at various symmetry points. The $\mathrm{K}$ symmetry point is chosen here because it is the least straightforward.

Consider the Bloch function that describes the sinusoidal nature of atomic orbitals in a repeating lattice:

$\psi(r)=e^{i k \cdot r} u(r)$

In this equation, $k$ is the crystal wave vector and $r$ is the position of the atom. The phase of an orbital can be described by the exponential term $k \cdot r$. This exponential term is the dot product of the crystal wave vector of a certain symmetry point and the vector that describes the orbital's location in real space. In the main text of this paper, the orbital phase given by $k \cdot r$ is referred to as $\theta$ such that the Bloch function becomes:

$\psi(r)=e^{i \theta} u(r)$

Let's calculate the phases of orbitals in adjacent unit cells at the $\mathrm{K}$ symmetry point. The crystal wave vector of the $\mathrm{K}$ symmetry point is given in equation (4). Now consider the following real space layer of hexagonal unit cells. In this figure, four atomic sites have been labeled as 1, 2, 3, and 4. These sites represent the locations of identical atoms in adjacent unit cells. The phase of each of these orbitals can be calculated by taking the dot product of the crystal wave vector at the $\mathrm{K}$ symmetry point and the vector that describes that atom's position in real space. 


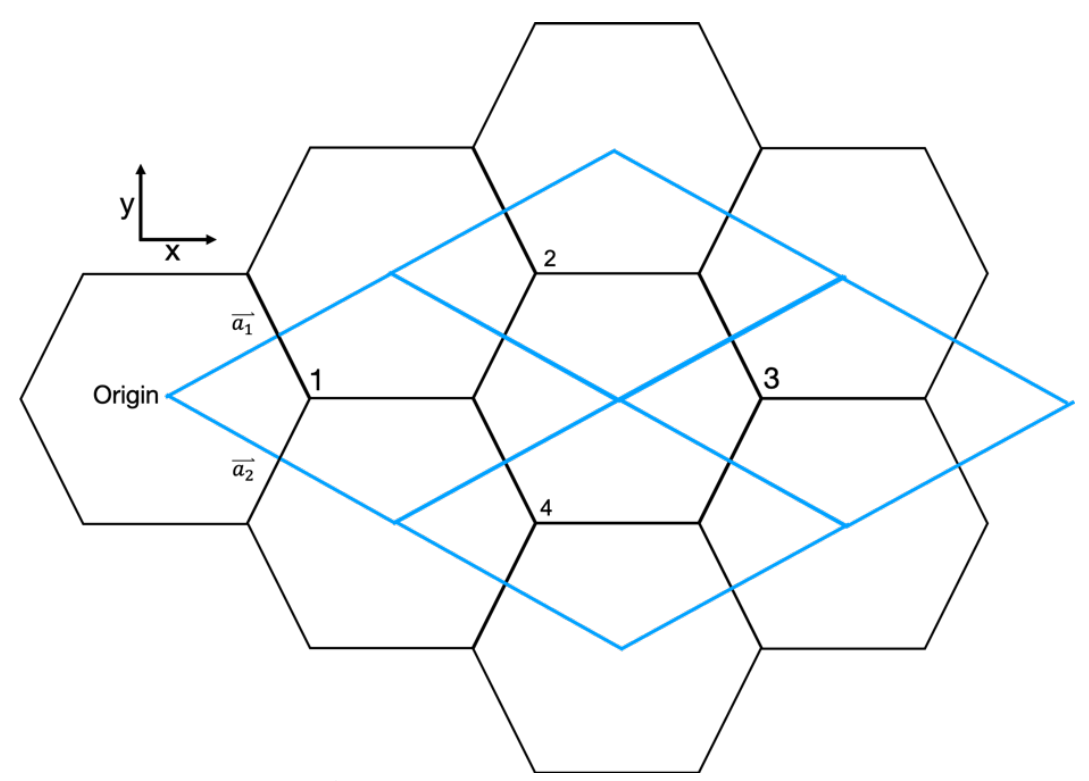

Figure S4. Real space atomic positions of equivalent atoms in adjacent unit cells, overlaid with unit cell lattice vectors.

For example, consider atom 1. As derived previously, its real space position $r$ can be described by the vector: $\overrightarrow{r_{1}}=\overrightarrow{c_{1}}=\frac{a}{\sqrt{3}} \hat{x}+0 \hat{y}$

This vector describes the atom's position relative to the origin point, as shown in Figure S1 earlier in this document. Multiplying this vector by the crystal wavevector at the $\mathrm{K}$ symmetry point will yield the phase of this orbital:

$$
\vec{K} \cdot \overrightarrow{r_{1}}=\frac{2 \pi}{\sqrt{3} a} \hat{k}_{x} \cdot \frac{a}{\sqrt{3}} \hat{x}+\frac{2 \pi}{3 a} \hat{k}_{y} \cdot 0 \hat{y}=\frac{2 \pi}{3}=120^{\circ} .
$$

This indicates that atom 1 has a phase of $120^{\circ}$. Now consider the phase of atom 2 . It is exactly one $\overrightarrow{a_{1}}$ vector away from atom 1 in real space. Since the position of atom 1 can be described by the vector $\overrightarrow{c_{1}}$, the position of atom 2 can be described by the sum of vectors $\overrightarrow{c_{1}}$ and $\overrightarrow{a_{1}}$ :

$$
\overrightarrow{r_{2}}=\overrightarrow{a_{1}}+\overrightarrow{c_{1}}=\left(\frac{a \sqrt{3}}{2}+\frac{a}{\sqrt{3}}\right) \hat{x}+\left(\frac{a}{2}+0\right) \hat{y}=\frac{5 a \sqrt{3}}{6} \hat{x}+\frac{a}{2} \hat{y}
$$

As with atom 1 , this resultant vector can again be multiplied by $\vec{K}$ to obtain the orbital phase of atom 2:

$$
\vec{K} \cdot \overrightarrow{r_{2}}=\frac{2 \pi}{\sqrt{3} a} \hat{k}_{x} \cdot \frac{5 a \sqrt{3}}{6} \hat{x}+\frac{2 \pi}{3 a} \hat{k}_{y} \cdot \frac{a}{2} \hat{y}=2 \pi=360^{\circ}
$$

This shows that the phase of orbital 2 is $240^{\circ}$ out of phase with that of atom 1 , or $120^{\circ}$ out of phase in the opposite direction. This calculation can be repeated for atom 3. To obtain the real space position of atom $3, \overrightarrow{a_{2}}$ is added to the position of atom 2, so that the net position of atom 3 is:

$$
\overrightarrow{r_{3}}=\overrightarrow{c_{1}}+\overrightarrow{a_{1}}+\overrightarrow{a_{2}}
$$

Multiplying this result by the $\mathrm{K}$ vector yields:

$$
\vec{K} \cdot \overrightarrow{r_{3}}=\frac{8 \pi}{3}=480^{\circ}
$$


This indicates that this orbital is in phase with atom 1 (out of phase by a value of $360^{\circ}$ indicates identical phasing), but out of phase with atom 2 by $120^{\circ}$. Finally, the same calculation may be performed for atom 4 . In order to reach atom 4 in real space, vector $\overrightarrow{a_{2}}$ is added to the position of atom 1 . This shows that the position of atom 4 is:

$$
\overrightarrow{r_{4}}=\overrightarrow{c_{1}}+\overrightarrow{a_{2}}
$$

Multiplying this result by the crystal wave vector $\vec{K}$ yields:

$$
\vec{K} \cdot \overrightarrow{r_{4}}=\frac{4 \pi}{3}=240^{\circ}
$$

This indicates that atom 4 is $120^{\circ}$ out of phase with atoms 1,2 , and 3 , as is expected at the $\mathrm{K}$ point. This phase calculation methodology can be more generally applied to find the orbital phases at other real space atomic positions, or at different symmetry points in the first Brillouin zone.

\section{DFT calculation method}

The density functional theory (DFT) calculations were done on the Vienna Ab Initio Simulation Package (VASP) ${ }^{1,2}$, using Perdew-Burke-Ernzerhof (PBE) ${ }^{3}$ functionals implemented in a projector augmented wave (PAW) method ${ }^{4}$, using a $12 \times 12 \times 6 \Gamma$-centered $\mathrm{k}$-mesh ${ }^{5}$ and a plane-wave cut-off of $350 \mathrm{eV}$ for relaxations. Initial lattice structures were obtained from MaterialsProject ${ }^{6}$ and were fully relaxed until the forces on all atoms are less than $10^{-5} \mathrm{eV} / \mathrm{A}$. Once the structure was relaxed, we increased the k-point density to $40 \times 40 \times 20$ with a tetrahedron smearing method to calculate band energy with better accuracy for reciprocal space integration in BoltzTraP ${ }^{7}$. The necessary derivatives were then calculated on a Fast Fourier Transform (FFT) grid 15 times as dense. The visualization of Fermi surface were performed with $\mathrm{XCrySDen}{ }^{8}$, where the band energies were calculated on a k-mesh composed of 27 evenly spaced $k$-points along $k_{x} / k_{y} / k_{z}$ axes.

\section{Supporting References}

(1) Kresse, G.; Hafner, J. Ab Initio Molecular Dynamics for Liquid Metals. Phys. Rev. B 1993, 47 (1), 558.

(2) Kresse, G.; Hafner, J. Ab Initio Molecular-Dynamics Simulation of the Liquid-Metal-AmorphousSemiconductor Transition in Germanium. Phys. Rev. B 1994, 49 (20), 14251.

(3) Perdew, J. P.; Burke, K.; Ernzerhof, M. Generalized Gradient Approximation Made Simple. Phys. Rev. Lett. 1996, 77 (18), 3865.

(4) Blöchl, P. E. Projector Augmented-Wave Method. Phys. Rev. B 1994, 50 (24), 17953.

(5) Monkhorst, H. J.; Pack, J. D. Special Points for Brillouin-Zone Integrations. Phys. Rev. B 1976, 13 (12), 5188.

(6) Jain, A.; Ong, S. P.; Hautier, G.; Chen, W.; Richards, W. D.; Dacek, S.; Cholia, S.; Gunter, D.; Skinner, D.; Ceder, G.; et al. Commentary: The Materials Project: A Materials Genome Approach to Accelerating Materials Innovation. APL Mater. 2013, 1 (1), 011002.

(7) Madsen, G. K. H.; Singh, D. J. BoltzTraP. A Code for Calculating Band-Structure Dependent Quantities. Comput. Phys. Commun. 2006, 175 (1), 67-71.

(8) Kokalj, A. XCrySDen-a New Program for Displaying Crystalline Structures and Electron Densities. J. Mol. Graph. Model. 1999, 17 (3-4), 176-179. 